\title{
Application of Electrodynamic Theory on Quantum Hall Effect
}

\author{
Saleem Iqbal1, Muhammad Zafar², Farhana Sarwar1,3, Syed Mohsin Raza4, \\ Muhammad Afzal Rana ${ }^{2}$ \\ ${ }^{1}$ Department of Mathematics, University of Balochistan, Quetta, Pakistan \\ ${ }^{2}$ Department of Basic Sciences, Riphah International University, Islamabad, Pakistan \\ ${ }^{3}$ Department of Mathematics, F. G. Girls Degree College, Quetta, Pakistan \\ ${ }^{4}$ Department of Physics, University of Balochistan, Quetta, Pakistan \\ Email: saleemiqbal81@yahoo.com,capt_zafar@yahoo.com,f_saleem10@yahoo.com, \\ smraza7@yahoo.com
}

Received 9 February 2016; accepted 13 May 2016; published 16 May 2016

Copyright (C) 2016 by authors and Scientific Research Publishing Inc.

This work is licensed under the Creative Commons Attribution International License (CC BY).

http://creativecommons.org/licenses/by/4.0/

(c) (i) Open Access

\begin{abstract}
The quantum electrodynamic (QED) behaviour is studied for quantum Hall effect (QHE). Quantum theory with conjecture of fractional charge quantization (quantum dipole moment), eigenfunctions for fractional charge quantization at the surface of a twisted and twigged electron quanta and above its surface, fractional Fourier transform and Hermite function for fractional charge quantization is developed. With energy eigen value equation for QHE and with energy operator on an eigenfunction of a twisted and twigged electron quanta, the corresponding eigenfunctions are normalized with Schrodinger's quantum wave mechanical equation for electric scalar and magnetic potentials, respectively (QED behavior). The fractional electric and magnetic fields with their corresponding potentials for the quantized fractional states in semiconducting hereto structures are theoretically calculated. Such mathematical expressions are in good agreement with experimental results of Nobel Prize winning scientists Klitzing, Haroche, Peter and Gruebber. Our results can also explain the hybridized states of orbits with emphasis on sigma and pi bonding and their corresponding antibonding orbitals as a manifestation of electrophilic and nucleophilic chemical reactions.
\end{abstract}

\section{Keywords}

Fractional Charge Quantization, Fractional Fourier Transform, Quantum Hall Effect, Quantum Electrodynamics, Electron Quanta String, Twisted and Twigged Electron Quanta

\section{Introduction}

Von Klitzing in the year 1987 won the Nobel Prize for physics on deciphering quantum Hall effect in heteoro-

How to cite this paper: Iqbal, S., Zafar, M., Sarwar, F., Raza, S.M. and Rana, M.A. (2016) Application of Electrodynamic Theory on Quantum Hall Effect. World Journal of Condensed Matter Physics, 6, 87-94. 
structures (sandwich like) semi conductors [1]. With this discovery, Van Wees et al. [2] reported "quantum conductance" in a two dimensional electron gas of GaAs-AlGaAs heteorostructure. Serge Haroche and David J. Wineland produced exciting experimental results on "individual quantum systems", without destroying the particle or quanta which led for them the Nobel prize winning award in physics 2012 "for ground-breaking experimental methods that enable measuring and manipulation of individual quantum systems” [3] [4]. To our understanding, the cavity quantum electrodynamics is a manifestation of fractional charge quantization or quantum dipole or quantum multi-pole moments [5]-[7]. We recently proposed with our conjuncture of fractional charge quantization, the measuring techniques of quantum behaviour for electric resistivity, dielectricity, giant magneto resistances (GMR) [8], Hall effect and conductance [9].

A new theory describing how charge being a constant physical entity on an electron in the momentum space is fractionally quantized, is presented [10] [11]. The eigen functions for a free electron quanta, twisting and twigging effects, at the surface, above the surface and inside the surface for the resuting electron quanta sring and twigs (sub-quanta) are determined [12]. With theory of fractional charge quantization for a free electron quanta above the surface, the shape of eigen functions, the energy eigen values and the quantum scattering for absorption in the matter are studied [13]. We conjuncture that the twisted and twigged electron quanta which appears in the form of an electron quant string with twigs (sub-quanta) on its lateral surface will be fractionally quantized in heterostructures semi conductors, as a consequence of which, quantum Hall effect due to fractional electric fields is observed with a gap of quantum Hall resistance, i.e., $R_{q} \sim h / e^{2} \sim 25813 \Omega$ [9]. This is why quantum electrodynamic behaviour of fractionally quantized single or many electrons in the semiconducting heteorostructures is crucial especially for learning the behaviour of electric and magnetic fields, and their corresponding potentials.

The magnetoresistance in quantum Hall effect (QHE) is of two types, one is longitudinal and the other is transverse. The longitudinal magnetoresistance is associated with magnetic field parallel to the current. The excitonic quantized Hall state becomes maximum at the total Landau level with vanishing longitudinal component and overwhelming transverse magnetoresistance [14]. This shows that the giant magnetoresistance (GMR) in quantum Hall effect (QHE) is due to transverse component of magnetic fields. There is no electronelectron interaction in quantum Hall effect (QHE). We conjuncture that (GMR) is due to fractionalized electric fields and a manifestation of gyroscopic constant, $g^{2} / \hbar c$, i.e., $0.02 \leq g^{2} / \hbar c \leq 0.08$ [7]. The QHE is discussed in detail by Gievin and McDonald [15]. The preliminary equations of quantum electrodynamics (QED) in our calculations are considered [16] [17].

\section{Results and Discussions}

Saleem Iqbal et al. [9] obtain the eigen functions for a twisted and twigged electron quanta on its surface by using the fractional Fourier ransform (FRFT) of order $\alpha$ (rotational angle) [18],

$$
\psi_{n_{f}}=\mathrm{e}^{i n_{f} \alpha}=\left(\frac{\alpha}{\sqrt{\pi} 2^{n_{f}}}\right)^{\frac{1}{2}} .
$$

Equation (1) represents plane wave for a rotation vector $\alpha$ in radians, on the surface of a twisted and twigged electron quanta (almost a quanta electron string), with fractional quantum numbers, i.e.,

$$
0.1 \leq n_{f} \leq 0.9 \text { and } 0.17 \leq \alpha \leq 1.53 \text {. }
$$

The Hermite function for the fractional quantum states [9] is

$$
H_{n_{f}}(\xi)=2^{n_{f}} ; 0.1 \leq n_{f} \leq 0.9 \text {. }
$$

The normalized eigenfunction for a twisted and twigged electron quanta above its surface [12] is given below:

$$
\Psi(r, \phi)=\sum_{r=0}^{\infty} r^{2 n_{f}} \sqrt{\frac{\left(4 n_{f}+1\right)}{r_{e}^{4 n_{f}+1}}} \mathrm{e}^{ \pm i m \phi} .
$$

Equation (4) yields theoretical eigenvalues

$$
E_{n_{f}}(\xi)=\frac{\left(n_{f}+2\right)\left(n_{f}+1\right) \hbar^{2}}{2 \mu \sin ^{4} \theta} ; 0.1 \leq n_{f} \leq 0.9 .
$$


$\phi$, the azimuthal angle in Equation (4) is related to Gyroscopic constant, where as Equation (5) refers to fractional quantum scattering of energy profiles for reduced mass $\mu$ of an electron. The enegy profile at the surface of the twisted and twigged electron quanta is fractionally quantized while the energy above its surface is consumed for quantizing the fractional states and appeared in the form of partial waves. This is experimentally evidenced [5]. For a twisted and twigged electron quanta in semiconductor heteorostructures one would like to know the quantum electrodynamic behaviour, especially in this study for QHE.

Rewriting the equation of QHE [9], we have

$$
\begin{gathered}
{\left[-e \bar{E}_{Q x}-\omega_{c}(\hbar k)_{y}-\frac{(\hbar k)_{x}}{\tau}\right] \psi_{n f}=i \hbar \frac{\partial}{\partial t} \psi_{n_{f}}=i \hbar \frac{\partial}{\partial t} e^{i n_{f} \alpha}=-\hbar n_{f} \psi_{n_{f}} \frac{\partial \alpha}{\partial t}} \\
{\left[-e \bar{E}_{Q y}+\omega_{c}(\hbar k)_{x}-\frac{(\hbar k)_{y}}{\tau}\right] \psi_{n f}=-\hbar n_{f} \psi_{n_{f}} \frac{\partial \alpha}{\partial t}} \\
E_{o p} \psi_{n f}=-\hbar \frac{\partial}{\partial t}\left(\frac{\alpha}{\sqrt{\pi} 2^{n_{f}}}\right)^{\frac{1}{2}}=-\frac{i \hbar}{2 \psi_{n_{f}}} \frac{\partial \alpha}{\partial t}
\end{gathered}
$$

where $\alpha$ is rotational angle of FRFT in time-frequency plane, $\psi_{n_{f}}$ the energy profile for fractional quantum states, $\hbar k$ the crystal momentum of the electron, $\tau$ the relaxation time, $\omega_{c}$ the cyclotron frequency and $Q \equiv Q_{n_{f}}$, i.e., $Q_{n_{f}}=\sum_{n=0.1}^{0.9} q_{n_{f}}$. The Hamiltonian in Equations (6a) and (6b) can be made Hermitian provided we know the values of $x$ and $y$ and change the crystal momentum with their corresponding momentum operators. With Hermitian Hamiltonian operator, energy eigen values can be determined. The QED behaviour of a quantized twisted and twigged electron quanta in the semi conductor heteorostructurescan be envisaged with electric fields (fractionally quantized in QHE). Writing the electric filed, $\bar{E}(\bar{r}, t)$ which is quantized due to a twisted and twigged electron quanta in semi conducting heteorostructures [16] [17].

$$
\bar{E}(\bar{r}, t)=-\frac{\partial \bar{A}(\bar{r}, t)}{\partial t}-\bar{\nabla} \cdot \phi(\bar{r}, t)
$$

where $\bar{A}(\bar{r}, t)$ is the magnetic vector potential, $\phi(\bar{r}, t)$ the electric scalar potential and $\bar{\nabla} \cdot \phi(\bar{r}, t) \equiv \operatorname{div} \phi(\bar{r}, t)$. With twisted and twigged electron quanta in semiconductor hetreostructure, dipole radiations with helicon energy profiles on either or both sides are produced. Rewriting the Schrodinger's quantum wave mechanical equation for dipole radiations [16] [17].

$$
-\frac{\hbar^{2} \nabla^{2} \psi}{2 m_{e}}-\frac{i e \hbar}{m_{e}} \cdot \bar{A} \cdot \nabla \psi_{n_{f}}+\frac{e^{2}}{2 m_{e}} \bar{A}^{2}(\bar{r}, t) \psi_{n_{f}}-e \phi \psi_{n_{f}}=E \psi_{n_{f}}=i \hbar \frac{\partial}{\partial t} \psi_{n_{f}}
$$

where $\bar{A}(\bar{r}, t)$ in Equation (8) for dipole radiations, is:

$$
\bar{A}(\bar{r}, t)=\sqrt{\frac{\hbar}{2 \varepsilon_{0} V}} \varepsilon^{(\lambda)} \mathrm{e}^{-i(\overline{k r}-\omega t)}
$$

where $\varepsilon^{(\lambda)}$ the permittivity of the semiconducting heteorostructure, $k$ the wave vector bounded with in the matter, i.e., $k=\frac{\sqrt{2 m(E-V)}}{\hbar}$ and $\varepsilon_{o}$ are the permittivity of free space. Equation (7) in compliance with Equation(8) for dipole radiation yields

$$
\bar{E}^{2}(\bar{r}, t) \simeq 2 \omega^{2} \bar{A}_{o} \bar{A}_{o}^{+} \simeq \omega^{2}\left(\bar{A}_{o} \bar{A}_{o}^{+}+\bar{A}_{o}^{+} A_{o}\right)-\omega_{o}^{2}\left[\bar{A}_{0} \mathrm{e}^{2 i(\overline{k r}-\omega t)}+\bar{A}_{o}^{+} \mathrm{e}^{-2 i(\overline{k r}-\omega t)}\right] .
$$

The last term in Equation (10) is oscillatory with natural frequency $\omega_{o}$, i.e., $-\omega_{o}^{2}\left[A_{o} \mathrm{e}^{2 i(\bar{k}-\omega t)}+\bar{A}_{o}^{+} \mathrm{e}^{-2 i(\bar{k} r-\omega t)}\right]$ which is neglected in our calculations. The part of some energy above the surface of the twisted and twigged electron quanta with its equivalent frequency coincides with natural frequency of the sample (resonance or damped oscillation), as a consequence of which, the energy associated with resonance is dissipated in free space 
where as the remaining energy is used to quantize the twisted and twigged electron quanta [Equation (5) is applicable with Equation (4)]. Equation (10) becomes

$$
\begin{gathered}
\bar{E}^{2}(\bar{r}, t) \simeq 2 \omega^{2} \bar{A}_{o} \bar{A}_{o}^{+} \simeq 2 \omega^{2} \frac{\hbar}{2 \varepsilon_{0} \omega V} \varepsilon^{2^{(\lambda)}} \simeq \frac{\hbar \omega}{\varepsilon_{0} V} \cdot \varepsilon^{2^{(\lambda)}} \\
\Rightarrow \bar{E}(\bar{r}, t) \equiv \sqrt{\frac{\hbar \omega}{\varepsilon_{0} V}} \cdot \varepsilon^{(\lambda)} .
\end{gathered}
$$

In our case, we have fractional electric fields appearing due to fractional charge quantization on twigs (subquanta) which are at the lateral surface of an electron string quanta.

$$
\bar{E}(\bar{r}, t)=\frac{\bar{F}_{Q_{F}}}{Q_{F}}=\sqrt{\frac{\hbar v_{Q_{F}}}{\varepsilon_{o} V_{Q_{F}}}} \varepsilon^{(\lambda)} .
$$

Changing the $x$ - and $y$-components of crystal momentum, $\hbar k$ with their corresponding operators, i.e., $-i \hbar \frac{\partial}{\partial x}$ and $-i \hbar \frac{\partial}{\partial y}$ and putting Equation (7) in equation (6a) and (6b), we have

$$
\begin{gathered}
{\left[-\frac{\partial}{\partial t} \bar{A}_{Q_{f}(\bar{r}, t)}-\nabla_{x} \cdot \phi(\bar{r}, t)-\omega_{c}\left(-i \hbar \frac{\partial}{\partial t}\right)-\frac{1}{\tau}\left(-i \hbar \frac{\partial}{\partial x}\right)\right] \psi_{n_{f}}=-\hbar n_{f} \psi_{n_{f}} \frac{\partial \alpha}{\partial t}} \\
\Rightarrow\left[\left(\frac{i \hbar}{\tau}-\phi(\bar{r}, t)\right) \frac{\partial}{\partial x}+i \omega_{c} \hbar \frac{\partial}{\partial y}\right] \psi_{n_{f}}=\left[\frac{\partial}{\partial t} \bar{A}_{Q_{f}}(\bar{r}, t)-\hbar n_{f} \frac{\partial \partial \alpha}{\partial t}\right] \psi_{n_{f}} \\
\quad\left[-\frac{\partial}{\partial t} \bar{A}_{Q_{f}(\bar{r}, t)}-\nabla_{y} \cdot \phi(\bar{r}, t)+\omega_{c}\left(-i \hbar \frac{\partial}{\partial x}\right)-\frac{1}{\tau}\left(-i \hbar \frac{\partial}{\partial y}\right)\right] \psi_{n_{f}} \\
\Rightarrow\left[\left(\frac{i \hbar}{\tau}-\phi(\bar{r}, t)\right) \frac{\partial}{\partial y}-i \omega_{c} \hbar \frac{\partial}{\partial x}\right] \psi_{n_{f}}=\left[\frac{\partial}{\partial t} \bar{A}_{Q_{f}}(\bar{r}, t)-\hbar n_{f} \frac{\partial \alpha}{\partial t}\right] \psi_{n_{f}} .
\end{gathered}
$$

On comparison of Equations (13) and (14), we have

$$
\begin{aligned}
& {\left[\frac{i \hbar}{\tau}-\phi(\bar{r}, t)\right] \frac{\mathrm{d}}{\mathrm{d} x} \psi_{n_{f}}+i \omega_{c} \hbar \frac{\mathrm{d} \psi_{n_{f}}}{\mathrm{~d} y}} \\
& =\left[\frac{i \hbar}{\tau}-\phi(\bar{r}, t)\right] \frac{\mathrm{d} \psi_{n_{f}}}{\mathrm{~d} y}-i \omega_{c} \hbar \frac{\mathrm{d} \psi_{n_{f}}}{\mathrm{~d} x} .
\end{aligned}
$$

Equation (15) on rearrangement for $\frac{\mathrm{d} \psi_{n_{f}}}{\mathrm{~d} x}$ and $\frac{\mathrm{d} \psi_{n_{f}}}{\mathrm{~d} y}$ terms becomes

$$
\left[i\left(\omega_{c}+\frac{1}{\tau}\right) \hbar-\phi(\bar{r}, t)\right] \frac{\mathrm{d} \psi_{n_{f}}}{\mathrm{~d} x}=\left[i\left(\frac{1}{\tau}-\omega_{c}\right) \hbar-\phi(\bar{r}, t)\right] \frac{\mathrm{d} \psi_{n_{f}}}{\mathrm{~d} y} .
$$

$\mathrm{d} \psi_{n_{f}}$ on LHS and RHS of Equation (16) is canceled

$$
\begin{gathered}
\Rightarrow \frac{\mathrm{d} y}{\mathrm{~d} x}=\frac{i \hbar\left(\frac{1}{\tau}-\omega_{c}\right)-\phi(\bar{r}, t)}{i \hbar\left(\frac{1}{\tau}+\omega_{c}\right)+\phi(\bar{r}, t)} \equiv \frac{y}{x} \\
\frac{y}{x}=\frac{i \hbar\left(\frac{1}{\tau}-\omega_{c}\right)-\phi(\bar{r}, t)}{i \hbar\left(\frac{1}{\tau}+\omega_{c}\right)+\phi(\bar{r}, t)}
\end{gathered}
$$


Equation (17) on simplification yields

$$
\phi(\bar{r}, t)=\frac{i \hbar\left[\left(\frac{1}{\tau}-\omega_{c}\right)-\left(\frac{1}{\tau}+\omega_{c}\right) \frac{y}{x}\right]}{1+\frac{y}{x}} .
$$

Subtracting Equation (16) from itself for both LHS and RHS, we have

$$
\begin{gathered}
2\left(\frac{1}{\tau}+\omega_{c}\right) i \hbar \frac{\mathrm{d} v_{n_{f}}}{\mathrm{~d} x}=2\left(\frac{1}{\tau}-\omega_{c}\right) i \hbar \frac{\mathrm{d} \psi_{n_{f}}}{\mathrm{~d} y} \\
\Rightarrow \mathrm{d} x=\frac{\left(\frac{1}{\tau}+\omega_{c}\right) \mathrm{d} y}{\frac{1}{\tau}-\omega_{o}} \Rightarrow x=\frac{\left(\frac{1}{\tau}+\omega_{c}\right) y}{\frac{1}{\tau}-\omega_{o}} \\
\Rightarrow \frac{y}{x}=\frac{\left(\frac{1}{\tau}-\omega_{c}\right)}{\left(\frac{1}{\tau}+\omega_{c}\right)} .
\end{gathered}
$$

Putting Equation (19) in Equation (18) for determining the electric scalar potential due to a quantized twisted and twigged electron quanta in semiconductor heteorostructures, we get on simplification

$$
\phi(\bar{r}, t)=0
$$

Equation (20) shows that the scalar electric filed potential is zero for fractional charge quantization, i.e., for QHE

Adding Equation (16) from its self for both LHS and RHS, we get on simplification

$$
\left[2 i \hbar\left(\frac{1}{\tau}+\omega_{c}\right)-2 \phi(\bar{r}, t)\right] \frac{\mathrm{d} \psi_{n_{f}}}{\mathrm{~d} x}=\left[2 i \hbar\left(\frac{1}{\tau}-\omega_{c}\right)-2 \phi(\bar{r}, t)\right] \frac{\mathrm{d} \psi_{n_{f}}}{\mathrm{~d} y}
$$

$\mathrm{d} \psi_{n_{f}}$ on both sides are canceled in the above expression and putting $\phi(\bar{r}, t)=0$

$$
\begin{gathered}
\frac{\mathrm{d} y}{\mathrm{~d} x}=\frac{2 i \hbar\left(\frac{1}{\tau}-\omega_{c}\right)-2 \phi(\bar{r}, t)}{2 i \hbar\left(\frac{1}{\tau}+\omega_{c}\right)-2 \phi(\bar{r}, t)}=\frac{i \hbar\left(\frac{1}{\tau}-\omega_{c}\right)-\phi(\bar{r}, t)}{i \hbar\left(\frac{1}{\tau}+\omega_{c}\right)-\phi(\bar{r}, t)} \\
\frac{\mathrm{d} y}{\mathrm{~d} x}=\frac{\frac{1}{\tau}-\omega_{c}}{\frac{1}{\tau}+\omega_{c}} \Rightarrow \frac{y}{x}=\frac{\frac{1}{\tau}-\omega_{c}}{\frac{1}{\tau}+\omega_{c}}
\end{gathered}
$$

which is similar to Equation (19). Thus, the validity of $\phi(\bar{r}, t)=0$ is proved. Now, considering Equation (6c), i.e.,

$$
E_{o p} \psi_{n_{f}}=\frac{-i \hbar}{2 \psi_{n_{f}}} \frac{\partial \alpha}{\partial t} .
$$

On comparison of RHS of Equations (13) and (14), we can put the equality of RHS to the equality of the above expression:

$$
E_{o p} \psi_{n_{f}}=-\frac{i \hbar}{2 \psi_{n_{f}}} \frac{\partial \alpha}{\partial t}=\left[\frac{\partial}{\partial t} \bar{A}(\bar{r}, t)-\hbar n_{f} \frac{\partial \alpha}{\partial t}\right] \psi_{n_{f}}
$$




$$
E_{o p}=\frac{\partial}{\partial t} \bar{A}(\bar{r}, t)-\hbar n_{f} \frac{\partial \alpha}{\partial t}=\frac{-i \hbar}{2\left|\psi_{n_{f}}\right|^{2}} \frac{\partial \alpha}{\partial t} \cdot \psi_{n_{f}} .
$$

Using Parseval' formulas,. i.e.,

$$
\int_{-\infty}\left|\psi_{n_{f}}\right|^{2} \mathrm{~d} n_{f}=\int_{-\infty}\left|a_{n_{f}}(k)\right|^{2} \mathrm{~d} k
$$

on Equation (22) where $a_{n_{f}}(k)$ is a complex constant with $k=\frac{\sqrt{2 m(E-V)}}{\hbar}$, i.e., in $k$-space

$$
\frac{\mathrm{d} \bar{A}(\bar{r}, t)}{\mathrm{d} t}-\hbar n_{f} \frac{\mathrm{d} \alpha}{\mathrm{d} t}=-\frac{i \hbar}{2\left|a_{n_{f}}(k)\right|^{2}} \frac{\mathrm{d} \alpha}{\mathrm{d} t} \psi_{n_{f}} .
$$

The $\psi_{n_{f}}$ in Equation (23) with its complex conjugate (linear inverse operator for mirror reflection) is normalized to identity operator. Thus Equation (23) takes the shape

$$
\begin{gathered}
\frac{\mathrm{d} \bar{A}(\bar{r}, t)}{\mathrm{d} t}-\hbar n_{f} \frac{\mathrm{d} \alpha}{\mathrm{d} t}+\frac{i \hbar}{2\left|a_{n_{f}}(k)\right|^{2}} \frac{\mathrm{d} \alpha}{\mathrm{d} t}=0 \\
\frac{\mathrm{d} \bar{A}(\bar{r}, t)}{\mathrm{d} t}=\left(\hbar n_{f}-\frac{i \hbar}{2\left|a_{n_{f}}(k)\right|^{2}}\right) \frac{\mathrm{d} \alpha}{\mathrm{d} t}
\end{gathered}
$$

Integrating this expression with respect to time

$$
\begin{gathered}
\int_{0}^{t} \frac{\mathrm{d} \bar{A}(\bar{r}, t)}{\mathrm{d} t} \cdot \mathrm{d} t=\int_{0}^{t}\left(\hbar n_{f}-\frac{i \hbar}{2\left|a_{n_{f}}(k)\right|^{2}}\right) \frac{\mathrm{d} \alpha}{\mathrm{d} t} \cdot \mathrm{d} t \\
\bar{A}(\bar{r}, t)=\left(\hbar n_{f}-\frac{i \hbar}{2\left|a_{n_{f}}\right|^{2}}\right) \alpha(t) .
\end{gathered}
$$

For oscillatory effect in semi conductor hetreostructure the energy is dissipated, i.e.,

$$
\frac{i \hbar}{2\left|a_{n_{f}}\right|^{2}} \alpha(t)
$$

becomes negligible. Equation (25) becomes

$$
\bar{A}(\bar{r}, t) \simeq \hbar n_{f} \alpha(t) .
$$

Equation (26) shows that the magnetic vector potential for fractional quantized states depends on twisting angle $\alpha$ with its twisting time and that it is also related to quantum action in twigs (sub-quanta) for QHE in semiconductor hetreostructure. Knowing $\phi(\bar{r}, t)$ and $\bar{A}(\bar{r}, t)$, it is easy to calculate the $\bar{E}(\bar{r}, t)$ and $\bar{B}(\bar{r}, t)$, which are the eigen values of QHE provided the QED behaviour is accounted for fractional quantization of electric fields and indeed the fractional charge quantization due to twisted and twigged electrons quanta in semiconductor heteorostructure. Using Equation (10) for QED behaviour of fractional charged quantization on twigs,. i.e.,

$$
\bar{E}^{2} \cong 2 \omega^{2} \bar{A}^{+}(\bar{r}, t) \bar{A}(\bar{r}, t)
$$

where

$$
\bar{A}^{+}(\bar{r}, t) \simeq-\hbar n_{f} \alpha(t)
$$


[from Equation (26)]

$$
\begin{gathered}
\left|\bar{A}^{+}(\bar{r}, t) \bar{A}(\bar{r}, t)\right| \cong\left|\hbar n_{f} \alpha(t)\left(-\hbar n_{f} \alpha(t)\right)\right|=\left|-\hbar^{2} n_{f}^{2} \alpha^{2}\right|=\hbar^{2} n_{f}^{2} \alpha^{2} \\
\bar{E}^{2}(\bar{r}, t) \cong 2 \omega^{2} \bar{A}^{+}(\bar{r}, t) \bar{A}(\bar{r}, t) \cong 2 \omega^{2} \hbar^{2} n_{f}^{2} \alpha^{2} \\
\bar{E}(\bar{r}, t) \cong \sqrt{2} n_{f} \alpha \omega \hbar .
\end{gathered}
$$

Equation (27) shows the quantization of fractional electric field for $0.1 \leq n_{f} \leq 0.9$ and $0.17 \leq \alpha \leq 1.53$. $\bar{E}(\bar{r}, t)$ also depends on frequency, $\omega$ of the fractional quantized states, i.e., twigs on the lateral surface of an electron quanta string. Equation (3) is self explanatory to reflect that twigs(sub-quant) although integrated on an electron quanta string are oscillating with different frequencies [pl see Equation (3)]. This confirms the fact that the fractional quantization is both adiabatic and harmonic time-dependent perturbation. Equation (27) can be correlated with Equation (12) for electric fields due to fractional charge quantization.

$$
\bar{E}_{Q_{f}}(\bar{r}, t) \cong \sqrt{2} n_{f} \alpha \omega_{Q_{f}} \hbar \cong \sqrt{\frac{\hbar v_{Q_{f}}}{\varepsilon_{o} V_{Q_{f}}}} \cdot \varepsilon^{(\lambda)}
$$

where $v_{Q_{f}}$ is the frequency of twigs (sub-quanta), $V_{q_{f}}$ the potential energy of twigs on an electron quanta string and $\varepsilon^{(\lambda)}$ the permittivity of semiconducting heteorostructure. $\lambda$ can have two values, i.e., 1 and 2 for monopoles and dipoles respectively. Now, we calculate the magnetic fields due to each twig on an electron quanta string. Using Maxwell's equation:

$$
\begin{gathered}
\text { Curl } \bar{E}=\bar{\nabla} \times \bar{E}=-\frac{\partial B}{\partial t} \\
\int \mathrm{d} B=-\int(\bar{\nabla} \times \bar{E}) \mathrm{d} t=-\int\left(\frac{\partial}{\partial r} \times \bar{E}\right) \mathrm{d} t .
\end{gathered}
$$

Putting Equation (27) in Equation (29), we have

$$
\begin{aligned}
& \bar{B}=-\int_{0}^{t}\left(\frac{\partial}{\partial r} \times \sqrt{2} n_{f} \alpha \omega_{Q_{f}} \hbar\right) \mathrm{d} t \\
&=-\int_{0}^{t}\left(\frac{\mathrm{d}}{\mathrm{d} r}\left(\sqrt{2} n_{f} \alpha \omega_{Q_{f}} \hbar \sin \theta\right)\right) \mathrm{d} t \\
& \cong-\sqrt{2} n_{f} \omega_{Q_{f}} \hbar \sin \theta \int_{0}^{t} \frac{\mathrm{d} \alpha}{\mathrm{d} r} \cdot \mathrm{d} t \cong-\sqrt{2} n_{f} \omega_{Q_{f}} \hbar \sin \theta \frac{\mathrm{d} \alpha}{\mathrm{d} r} \cdot t \\
& \cong-\sqrt{2} n_{f} \omega_{Q_{f}} \hbar \sin \theta \cdot t \cdot \frac{\mathrm{d} \alpha}{\mathrm{d} r} ; \quad \theta=\omega t \Rightarrow t=\frac{\theta}{\omega} \\
& \bar{B} \cong-\sqrt{2} n_{f} \omega_{Q_{f}} \hbar \sin \theta \cdot \frac{\theta}{\omega_{Q_{f}}} \cdot \frac{\mathrm{d} \alpha}{\mathrm{d} r} \cong-\sqrt{2} n_{f} \hbar \theta \sin \theta \bar{\nabla}_{r} \alpha \\
& \quad \bar{B}(\bar{r}, t) \cong-\sqrt{2} n_{f} \hbar \theta \sin \theta \bar{\nabla}_{r} \alpha .
\end{aligned}
$$

The negative sign shows induced magnetic fields due to twisting and corresponding phase changes (energy changes) on twigs. Neglecting the negative sign, we have

$$
\bar{B}_{Q_{f}}(\bar{r}, t) \cong \sqrt{2} n_{f} \hbar \theta \sin \theta \bar{\nabla}_{r} \alpha .
$$

Equation (30) shows that

$$
\theta \sin \theta \bar{\nabla}_{r} \alpha
$$

follows the helical pattern for dipole radiations due to twisted magnetic fields for each of the twigs on the lateral surface of an electron string quanta in semiconducting hetreostructures for QHE. $\theta \sin \theta \equiv \omega t \sin \omega t$ which if multiplied by $\bar{\nabla}_{r} \alpha$ will corresponds to phase changes as a manifestation of change of $\alpha$ (rotation vector) with curvatures in space. The helical like dipole radiation from twigs due to their corresponding magnetic fields 
make them (twigs) to remain oscillatory. The oscillatory effect of twigs with their corresponding characteristic frequencies on an electron quanta string follows an adiabatic perturbation and is a manifestation of fractional charge quantization. The evidence of this phenomena is supported by the discovery of GMR by Albert Peter and Paul Gruebber and this led them to winning the Nobel prize in the year 2007 [8]. Similarly, the single electron tunneling across the interface state of a transistor will follow a helicon profile with each turn of the helix corresponding to fractional quantum states (spintronics). The fractional Hall electric fields are like pearls beaded in an electron quanta string and each string is connected to another electron string with twisting effects as a manifestation of QED behaviour. Thus, we have a quantum Garland beaded twigs (sub-quanta) on a single or many electron quanta strings.

\section{Conclusion}

With conjecture of fractional charge quantization, eigenfunctions for a bounded, stretched, twisted and twigged electron quanta are obtained. The fractional electric and magnetic fields at each of the twigs of a bounded, stretched, and twisted electron quanta, and the corresponding scalar electric and magnetic vector potentials for the quantized fractional states in semiconducting heterostructures are theoretically calculated and explained with obtained mathematical relations. Our results can also explain the hybridized states of orbits with emphasis on sigma and pi bonding and their corresponding antibonding orbitals as a manifestation of electrophilic and nucleophilic chemical reactions.

\section{References}

[1] Klitzing, V. (1987) Quantum Hall Effect in Heterostructure Semiconductors. American Physical Society News Letter.

[2] Van Wees, B.J., Van Houten, H., et al. (1988) Quantized Conductance of Point Contacts in a Two-Dimensional Electron Gas. Physical Review Letters, 60, 848-850. http://dx.doi.org/10.1103/PhysRevLett.60.848

[3] Haroche, S. and Kleppner, D. (1989) Cavity Quantum Electodynamics. Physics Today, 42, 24-30. http://dx.doi.org/10.1063/1.881201

[4] Haroche, S. (1995) Manipulating Quantum Fields with a Single Atom in a Cavity. The 7th International Symposium: Resonance Ionization Spectroscopy, 329, 30-35. http://dx.doi.org/10.1063/1.47571

[5] Yousaf, S., Raza, S.M., et al. (2008) Absorption of Radiant Energy in Water: A New Conjecture and Theory of Charge Quantization in Chromotized Water Samples. Science International Lahore. Pakistan, 20, 189-195.

[6] Rehman, F., Raza, S.M., et al. (2009) Quantum Theory of Dielectric and Its Applications to Dolomite of Balochistan, Pakistan. Science International Lahore. Pakistan, 21, 29-32.

[7] Jabeen, S., Raza, S.M., et al. (2012) Quantum Mechanical Analysis on Faujasite-Type Molecular Sieves by Using Fermi Dirac Statistics and Quantum Theory of Dielectricity. Journal of the Chemical Society of Pakistan, 28, 251-255.

[8] Fert, A. and Grnberg, P. (2007) Giant Magnetoresistances. APS News Letter, 16, No. 10.

[9] Iqbal, S., Sarwar, F., et al. (2016) Applications of Quantum Physics on Resistivity, Dielectricity, Giant Magneto resistance, Hall Effect and Conductance. WJCMP, 6.

[10] Iqbal, S., Sarwar, F., Raza, S.M. and Rehman, A. (2015) How Fractional Charge on an Electron in the Momentum Space is Quantized? ASRJETS, 14, 265-272.

[11] Iqbal, S. (2012) Analysis and Applications of Fractional Fourier Transform. Unpublished PhD Thesis, University of Balochistan, Quetta, Pakistan.

[12] Iqbal, S., Sarwar, F. amd Raza, S.M. (2016) Eigenfunctions for a Quantum Wire on a Single Electron at Its Surface and in the Quantum Well with Beaded Fractional Quantized States for the Fractional Charges. WJAMP, 4, 320-327.

[13] Yousaf, S., Raza, S.M. and Ahmed, M.A. (2008) Newly Developed Recursive Relationship for Fractional Quantum States and Associated Energy Eigen Values. Science International (Lahore), 20, 255-260.

[14] Kellogg, M., Spielman, I.B., et al. (2002) Observation of Quantized Hall Drag in a Strong Correlated Bilayer Electron System. Physical Review Letter, 88, 126801-126804. http://dx.doi.org/10.1103/PhysRevLett.88.126804

[15] Gievin, S.M. and Macdonald, A.H. (1997) Prospective in Quntum Hall Effects. Wiley, New York.

[16] Gaseoroweiz, S. (2003) Quantum Mechanics. 3rd Edition, Wiley, New York.

[17] Griffth, D.J. (2005) Introduction to Quantum Mechanics. 2nd Edition, Pearson Education.

[18] Almeida, L.B. (1994) The Fractional Fourier Transform and Time-Frquency Representations. IEEE Transactions on Signal Processing, 42, 3084-3091. http://dx.doi.org/10.1109/78.330368 